\title{
Knowledge representation in our systems
}

\author{
V. Aggelis \\ Piraeus Bank SA, Electronic Banking Division, Greece
}

\begin{abstract}
Data utilization has become a critical point in business success. Since enormous data volumes are gathered from companies, institutions and organizations, there has arisen a need for taking advantage of the collected information. Nowadays, many models and processes can be found, worldwide, for data management and utilization. "Knowledge representation in our systems" (KRIOS) is not only another model. KRIOS is also a new philosophy which targets bringing a revolution in the business environment. Our method's scope is to emphasize the human factor related to the process. KRIOS includes three parallel layers.

The first is a step by step methodology which describes all steps from gathering data until gaining competitive advantages from knowledge, using data mining techniques. The whole process is iterative and is executed continuously. This is the corollary of data texture and data characteristic.

The second one is the human factor layer. All steps are reflected in human roles and attributes. The KRIOS initiation point is not the first step of its methodology. The starting point is top management. Top managers' faith and support of KRIOS are prerequisites for its successful adoption. As we stated in our work there is the Lamp Example, which describes top management's contribution to our model.

Finally, the third layer refers to the knowledge and business level. Each step is connected with a piece of knowledge and business process. All layers, the methodology layer, human role layer and knowledge and business layer, are strongly interconnected.

Taking into consideration the human factor is an innovative approach. Well established models, theories or methodologies are good enough but all of them are applied by people. People are those who make them successful and useful. KRIOS is a new trend, a multi-tool, a leading decision support system and it will succeed, if it manages to become a siege ram in knowledge and in business process problems. KRIOS revolution depends on humans. Business benefits realization from data utilization and, mostly, faith in its contribution are the basic pylons of our philosophy. In addition KRIOS deals with customers and one of its aims is to also return gain and benefits to them.
\end{abstract}

Keywords: data utilization, data mining, knowledge, decision support. 


\section{Introduction}

All business organizations are much more interested in data management and data utilization than they were in past decades. Enormous data volumes spotlight the need for its utilization. Everyone can find many models for such usage in international bibliography. The scope of all those models is to organize the data management process in separated and well understood steps in order to contribute to business process optimization and reengineering

However, many of them don't manage to fit to modern business needs. That lack of fitting depends on the human factor. The human factor is the crucial point. Humans are responsible for acting in accordance with models, methodologies and theories. Humans are the executive arm. So, even a well established procedure cannot work properly, if it interacts badly with executives.

KRIOS apart from its step description shows all human roles and their contribution to a model, in order for knowledge utilization to stand as a really useful and essential tool with multiple gains.

The name KRIOS comes from the initials of the phrase "Knowledge Representation in Our Systems" and was established in 2005. It evolved in WINBANK, which is the electronic banking division of Piraeus Bank. First it was implemented as a statistical information system and after a short period was transformed to an integrated knowledge utilization platform. Knowledge representation, data mining, decision support and proposed actions are part of WINBANK KRIOS. Simple reporting and graph reading have no actual value if they are not accompanied with decisions and actions. Those characteristics are the success factors and they contribute to its whole acceptance by WINBANK internal users.

Our big challenge is data and knowledge utilization. KRIOS is our vision. We want our vision to become a real business procedure. So our intention is to make KRIOS known worldwide in the business and academic communities.

In the next sections we describe our model and our integrated data utilization platform. Section 2 contains the KRIOS model, while in section 3 the WINBANK goal is described. Section 4 contains the WINBANK KRIOS system and section 5 describes the impacts of our integrated platform. Finally, in section 6 we present the next steps in our system.

\section{KRIOS model}

The KRIOS process consists of the following steps

1. Collect and explore data

2. Understand and organize data

3. Design data repository and Extract-Transform-Load process

4. Get information

5. Find hidden knowledge

6. Take decisions

7. Make actions

8. Take (competitive) advantages

The KRIOS model [17] is shown in Figure 1. 


\section{KRIOS PROCESS MODEL}

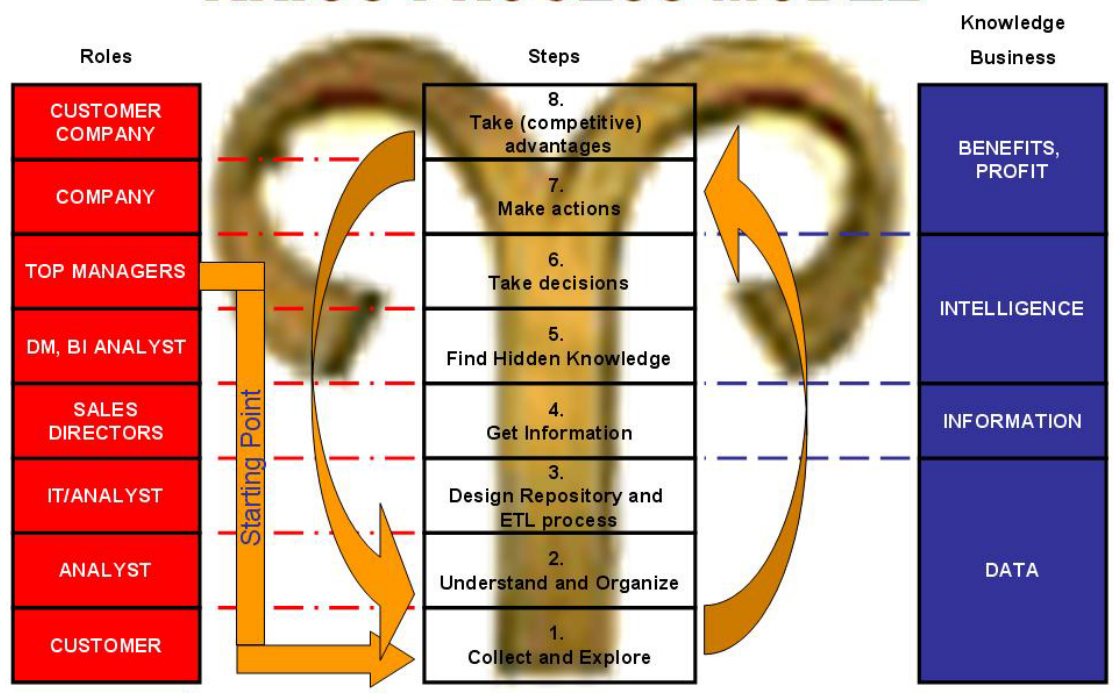

Figure 1: KRIOS model.

The whole process is iterative and runs continuously. This is the corollary of data texture and data characteristic. New data are added; current data are transformed or changed, so its management and utilization is a consecutive stepwise process. A static data management process is a fatal flaw for any organization.

Process steps are represented in the Business and Knowledge Layer. The three first steps relate to the raw data layer.

Data collection means specific definition of collected information and data repository identification, where the collected data are stored. Data exploration consists of a record of possible missing information and data intercross.

In order to understand data having business process knowledge and a deep experience of it is prerequisite. Also analysis skills are necessary for the understanding of collected data repositories.

The final step is represented as raw data in the business and knowledge layer, concerning the definition of entities and their attributes. It is very important to set the process for data extraction, data transformation, data cleansing and data loading.

The fourth step of KRIOS is referred to the information layer. Before having information representation it is critical to manage missing values, which inflect in many cases the results. After that task, information can be represented as a report or graph. Nowadays there are more sophisticated representation forms, like Key Performance Indicators (KPI) or Visualization.

The next layer is the intelligence one. In order to make your business intelligent, hidden information and patterns must be found, using at most data 
mining techniques. Data mining [1] would advance intelligence in decision making. There are many practices that could help to take decisions, such as advanced or predictive analytics.

The last layer is related with benefits and profit that are gained from the whole process. Speaking about gains, it is important to emphasize that gains are both for companies and their customers.

Discrete human roles are defined in the KRIOS model. The human factor [16] is the innovative part of the model. All roles have the same importance, but two of them are most crucial. Those roles are:

- Customer: Customer is the initial and better data source. Any customer could be internal (organization staff) or external. Customer transactions produce huge data volumes daily. Customers buy, pay, deposit, order, register, allocate, cancel, navigate, have needs, have opinions, work, live, marry, study and make many other actions that have new data production or current data transformation as results. At the same time customers are recipients of knowledge and benefits from the KRIOS process.

- (Business) Analyst: Analysts must be qualified with business process knowledge and data manipulation skills.

- IT staff: The contribution of IT staff is necessary because they are the most appropriate to design in cooperation with business analysts the data repository and implement it. Also they could help in the ETL process design and its automated operation.

- Sales Departments: Company's sales departments receive information about crucial metrics via different systems, like MIS, CRM, etc. In addition they receive clear and steady information for their customers, in order to plan the next actions.

- Directors: Information received by Directors differs than information got by sales departments. It is not so detailed, but contributes to a safe and real representation of business value. Directors belong to the decision-makers group.

- Data Mining or Business Intelligence Analyst: This specific analyst has to know data mining and business intelligence techniques and methods. It the second most crucial role because they are the ones who add value in the business chain by establishing models and patterns for hidden information.

- Top management: Executives, Presidents, General Directors are those who take the critical decisions for a company. Top management is the most crucial role, the foundation stone of KRIOS. They are the starting point. If they don't believe in KRIOS capabilities there is no chance to take part in the KRIOS revolution.

- Company: with this abstract notion we mean all the company's staff that are responsible for execution of the business plan and actions. A company's shareholders also participate in this notion. They are those who gain benefits from a company's revenues. 


\section{WINBANK goal}

WINBANK designed, implemented and established an innovative smart knowledge returning system [9]. As is shown in Figure 2, the system has dual goal. It serves WINBANK's business needs, but it also serves our customer.

Our viewpoint is that the internet banking channel is not only an easy and time-saving way for executing banking transactions, but it is also an interactive contact with the customer. So we take advantage from KRIOS in order to build a steady and healthy relationship with our users. As a first step we offer personalized services for each user. Moreover, taking into account many parameters, our internet banking service returns knowledge to the user during his/her navigation in our site.

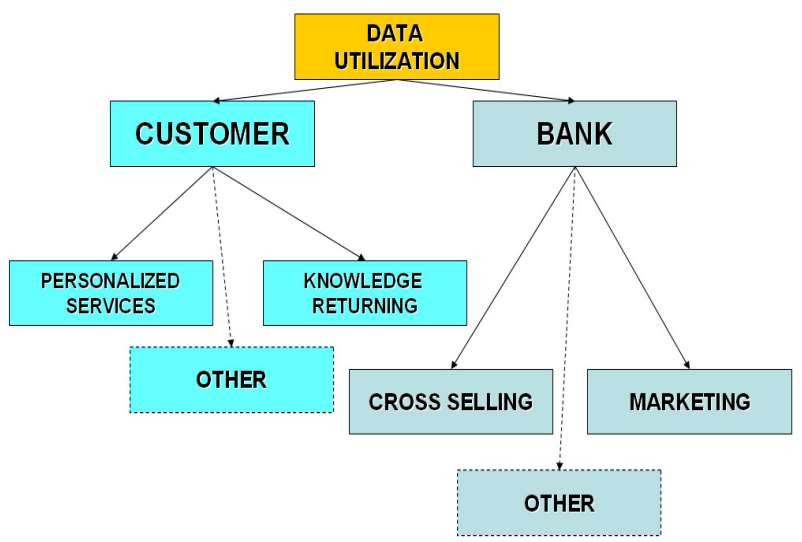

Figure 2: Our goal.

In order to implement the main functions of the system to serve our goal, there are many cooperating parts of KRIOS. These parts are:

- Data Warehouse: All electronic transactions (both informational and financial), all transactions from other channels (cashier, ATMs, Automatic Payment Systems, etc.) and the web log from the secure internet banking site are stored in the Data warehouse. All this information is updated on a weekly basis.

- Modeler: Modeler is the subsystem which consist data mining, business intelligence, predictive analytics and ETL (Extract-Transform-Load) tools. This subsystem is responsible for data processing. In addition, the modeler creates and educates models and patterns, which, in most cases, is stored in the Knowledge Base.

- Knowledge Base: Our knowledge base is the main knowledge repository. Every single piece of information represented to users is exported from this base. The Knowledge base is updated from the data warehouse, modeler and user's interaction. 


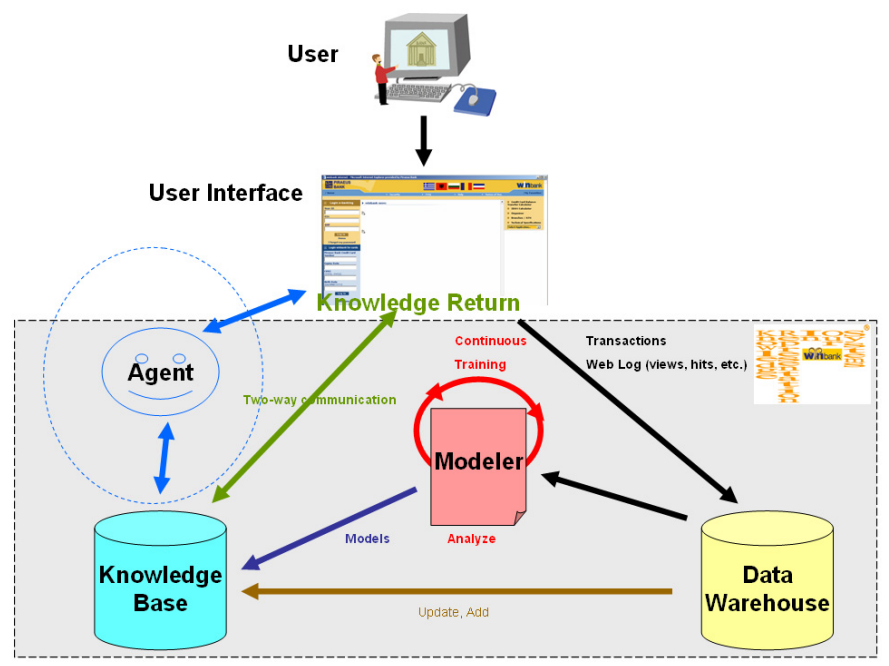

Figure 3: KRIOS diagram.

- Smart Agent (optional): This is an optional tool. Its usefulness is based on contributing in a more friendly and familiar interface.

Figure 3 describes the KRIOS infrastructure and operation diagram and how it acts for our users' privilege.

\section{WINBANK KRIOS system}

The whole system's topology is shown in Figure 4. There is a central KRIOS Data warehouse, which is loaded from various sources, such database servers, AS/400, ASCII files, etc. All the BI and data mining process is executed using algorithms from SPSS Clementine.

WINBANK KRIOS is available only to internal users of our division. For critical applications, like Internet Banking Fraud Detection, there is an authentication mechanism, in order to give someone access to it.

Our platform [12] is divided into four main sections. Each of them has a separate scope.

- MIS

- Fraud Detection

- Data Mining

- $\quad$ SecurID Management

Regarding MIS, there are 19 different applications so far, related to our services, products, customers and targets. The reporting capability is tremendous. There are thousands of reports, but few templates. For example, one transaction report template for Internet Banking produces 924 different reports. Apart from reporting, there are several other useful forms and graphs that help our managers and staff in their daily work. 


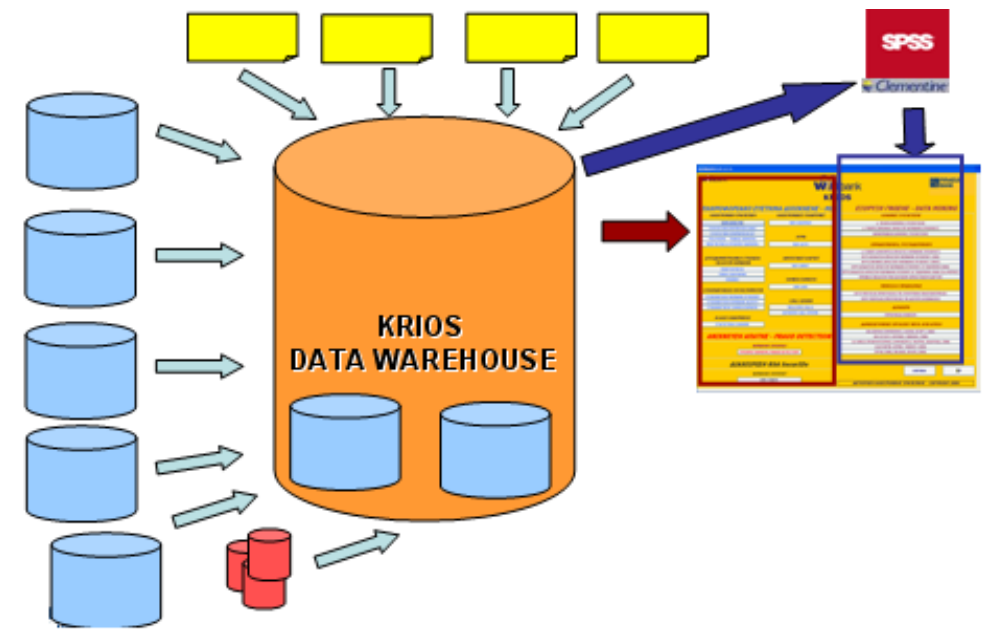

Figure 4: KRIOS topology.

SecurID Management is an internal workflow-like application which is used by our Operations, in order to take orders for tokens delivery and to check the stock of our SecurIDs, their statuses and staff like that with many enhanced reports.

The most interesting issue for WINBANK KRIOS is that it is developed, implemented, and updated in-house by one person. The whole project took about six months to implement and was up and running from the beginning of 2006.

\subsection{Data mining section}

Data Mining [4] has a main role in our model as well in our system. As we stated in section 3, our goal is to return knowledge to our end-users, but also to take competitive gains. So we have established various models, which are continuously educated.

A brief description of our models follows.

e-Trans Rules: This model $[10,11,14]$ gives us the association rules between electronic transactions. The detection of such relationships $[5,7,8]$ offers a bank a detailed analysis that can be used as a reference point for the conservation of the customer volume initially but also for approaching new customer groups (households, companies, freelancers) who conduct these payments in order to increase its customer base and profit.

e-Trans Customer Clustering: With this model [13], we focus on customers that verify e-Trans rules and find their demographic and transactional profile. Having basic profiles assists WINBANK to make cross-selling campaigns.

e-Churners: This model describes the e-churner profile. We call an e-churner every user who did not login to our internet banking service in the last four months. There are 3 distinct clusters [6] of e-churners. We called them 
218 Data Mining VIII: Data, Text and Web Mining and their Business Applications

1. One glance

2. Account, and

3. Double product

All of them conducted only informational transactions in the last 6 months before they became inactive users.

In Figure 5 the e-churner distribution is shown. The majority of them are Account e-churners (56\%). Figure 6 shows the gender distribution.

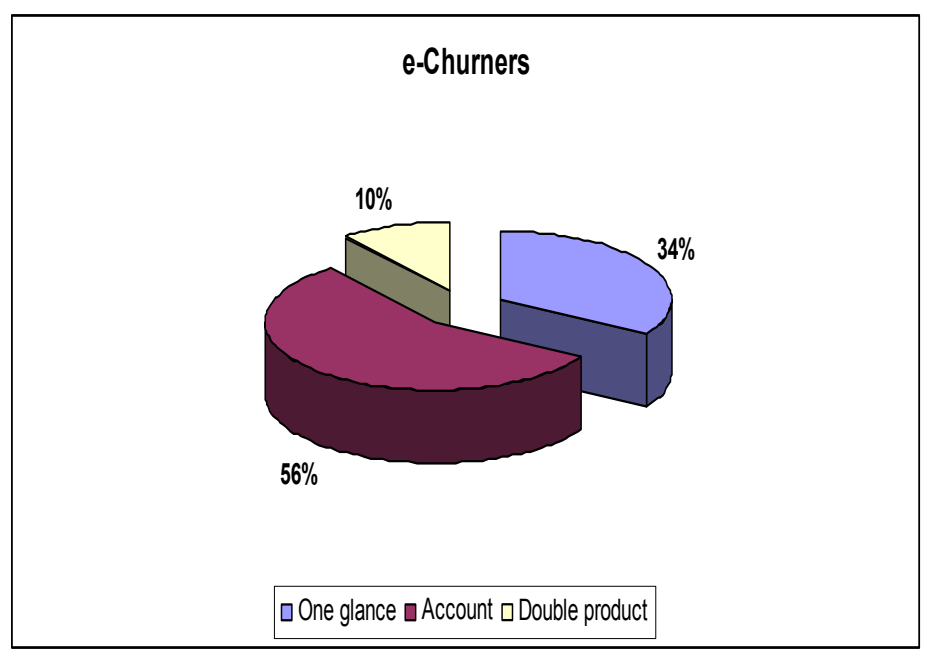

Figure 5: e-Churners distribution.

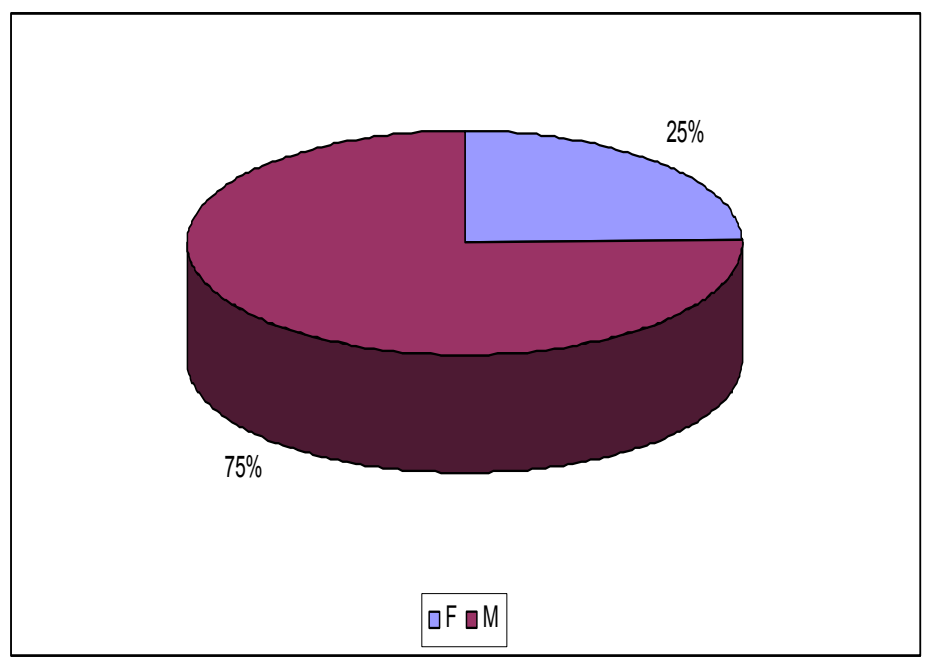

Figure 6: e-Churners gender distribution. 
Having that model educated, we can predict our future e-churners and make actions in order to reduce their leakage.

RFM Analysis: RFM [18] is a popular method in marketing. It is also important in other businesses like e-banking. Our RFM model scores all active customers and it is updated every semester. According to that model, we established 4 basic customer categories, TOP, BIG, MEDIUM and SMALL. We are in position to know our customer scores and their category, but the most interesting is that we can observe movements between those categories from semester to semester and focus on them, finding the related customer profiles. Also we know which customers are most probable to react to our campaigns.

RFM score prediction: There are two established and educated models for this scope. The first one is a formula aroused from linear regression and the second one is a classification and regression tree. Each of them helps us to predict with great possibility our customers RFM score, before the end of a semester.

BankPreBUs Rules: Bank Products of e-Banking Users Rules shows the association [2, 3] between bank products that have e-banking users. PIRAEUS BANK offers about 650 different products. With BankPreBUs rules we can find and state relationships between them in our e-banking users' community. This is very helpful because in some cases we find hidden associations that we did not ever have in mind. Those hidden associations drive us to enrich our electronic services with new types of transactions.

There are several other models. The scope of this paper is not to focus in detail on all of them, but to give an idea about how useful an intelligent system like WINBANK KRIOS is.

\subsection{Internet banking fraud detection}

A basic security feature of the WINBANK internet banking site is the two factor authorization. When a user conducts a risky financial transaction, in some cases, he/she has to put a one time password in order to end it. For user's convenience purposes not all transactions are characterized as risky. We call the following risky financial transactions [15]:

- Funds transfer between bank accounts.

- Funds transfer from a bank account to any other domestic or international bank account.

- Massive payments.

- Credit card payment. This payment refers only to credit cards issued by another bank.

Additionally every single user has a standard daily amount limit. That limit concerns only risky transactions and is the same for all users. If the total daily amount of a user's risky transactions exceeds their limit, then a one time password is needed for transaction completion.

The fact is that two factor authorizations discourage many candidate fraudsters. Nevertheless, they are not a panacea. Fraud never stops. Fraudsters override security framework, with particular steps. Firstly, they install a Trojan horse in a victim's computer. In most cases a Trojan horse is treated as a key 
logger. The next step is to logon to an internet banking site, pretending to be the real user. The fraudster finds out the customer's behavior. If the user often conducts fund transfers via internet banking they are not an appropriate victim. A fraud convenient victim is someone who uses internet banking only for informational purposes or someone who uses electronic services rarely.

In those cases, fraudster transfers daily amounts nearly to the daily limit. For such amounts a one time password is not necessary. The oblivious victim detects fraud after a long time. In this time frame the fraudster has made some fund transfers. If the time frame is longer than a week, the fraudster can take a remarkable amount into his/her account.

WINBANK took into consideration all parameters which lead to internet banking fraud. We established many detection rules. Apart from the initial ones, new rules are added, when analysis finds out suspect patterns and behaviors. Those rules are enhanced in an offline fraud detection system. The system is offline because of its database update process. New data are imported into the database in constant time frames, not in real time. For the time being there is no immediate need to upgrade the system in online mode.

Analysis, design and implementation of the system was done in-house. Due to the bank's data sensitivity, one of the prerequisites was an in-house set up and function of such a system. The pilot operation period proved the system's reliability, accuracy and success. Moreover the pilot period helped the bank scan the system for bugs, faults and defects. After that period, the fraud detection system began to operate in the production environment.

Suspect transactions are graduated. According to their risk, they are signed as high, medium and low risk. The probability of fraud is very low, less than $1 \%$. So the great majority of suspect transactions are not fraudulent. Nevertheless, bank obligates to search all suspect transactions. Apparently the final target is the online implementation of the above described system.

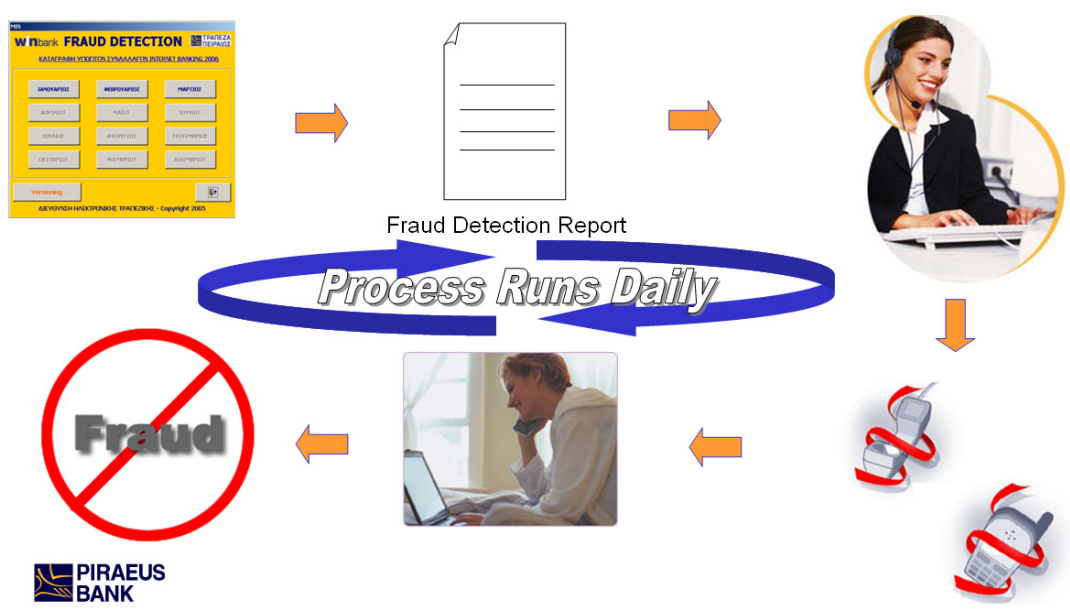

Figure 7: Daily fraud detection process. 
Special staff receive fraud detection reports. Those people undertake the mission to contact with customers. Communication with internet banking users is a crucial step of the whole process. Many of us consider this communication more important than the system itself. As we stated almost 99 in 100 suspect transactions are not fraudulent. So in 99 out of 100 cases the agent must not cause concern [15]. The agent has to talk with the customer calmly and friendly, indicating the bank's concern for them. There is no need to betray the basic reason for the call. The agent asks the customer some questions, reassuring the transactions' legitimacy. The main risk of contact is that there is a possibility to frighten the customer. In that case the customer becomes a more infrequent user, and maybe stops conducting internet banking transactions.

The whole process that is followed daily is shown in Figure 7.

When a fraud is reassured, then the bank takes all the necessary measures against fraudsters and protects and guarantees its customer deposits.

\section{Impacts}

We have already stated many privileges of our system. KRIOS offers the following advantages:

- Good knowledge of the relationships between different types of electronic transactions.

- Description and establishment of most popular internet transactions.

- The electronic services become more easily familiar to the public since specific groups of customers are approached, that use specific payment manners.

- The customer approach is well designed with higher possibility of successful engagement.

- The improvement of already offered bank services is classified as to those used more frequently.

- Redesign internet transaction structures for those which used rarely.

- Reconsidering of the usefulness of products exhibiting little or no contribution to the rules.

- Personalized menus through preference mining.

- Customer views returning information via the internet banking site.

On the other hand, the offline internet banking fraud detection system offers many benefits to both the bank and customers.

- Fraud detection system gives added value to e-banking. Especially, nowadays, where fraudsters' attacks are increased considerably in our country, such a system differentiates bank owners from other bank competitors.

- The bank takes the lead. Such in-house system implementations, which are set up for the customer benefit, are infrequent in the local market.

- The fraud detection system indicates the quality of e-banking services. Quality depends on a user friendly interface, on a full range of 
electronic transactions portfolio, but also depends on user protection and guarantees.

- A significant number of users have the sense of care and protection from their bank. This sense helps customer loyalty escalation.

- Official fraud victims are informed by the bank itself as soon as fraud is detected. Customers feel that their bank stands by them and that fact strengthens mutual relations.

\section{Future steps}

The next steps of the KRIOS model and system in Piraeus bank are:

1. Business Intelligence Strategy Establishment for the whole bank.

2. Foundation of a Business Intelligence unit independent from IT, with a horizontal view of all business units and processes.

3. An effort to reduce the fatal flows of Business Intelligence.

4. Piraeus bank KRIOS development and implementation.

5. WINBANK KRIOS incorporation in Piraeus bank KRIOS.

6. International KRIOS development and implementation.

\section{References}

[1] Hand D., Mannila H. \& Smyth P., Principles of Data Mining, The MIT Press, 2001

[2] Toivonen H., Discovery of Frequent Patterns in Large Data Collections, Technical Report A-1996-5, Department of Computer Science, University of Helsinki, 1996.

[3] Im K. \& Park S., A Study on Analyzing Characteristics of Target Customers from Refined Sales Data, APIEMS, 1999.

[4] Chen M., Han J. \& Yu P., Data Mining: An Overview from Database Perspective, IEEE Transactions on Knowledge and Data Engineering, 1997.

[5] Zaki M., Parthasarathy R., Li W. \& Ogihara M., Evaluation of Sampling for Data Mining of Association Rules, 7th Workshop Research Issues on Data Engineering, 1997

[6] Bradley P. \& Fayyad U., Refining Initial Points for K-Means Clustering, 15th International Conference on Machine Learning, 1998.

[7] Agrawal R., Mannila H., Srikant R., Toivonen H. \& Verkamo A.I., Fast discovery of associations rules, Advances in Knowledge Discovery and Data Mining, 1996

[8] Brin S., Motwani R. \& Silverstein C., Beyond Market Baskets: Generalizing Association Rules to Correlations, Proceedings ACM SIGMOD Conf. on Management of Data, 1997

[9] Aggelis V., A Smart Knowledge Returning System, 2nd International Conference on Business Management and Economics, 2006. 
[10] Aggelis V., Application and Diachronic Strength of e-Trans Association Rules, 5th IBIMA Conference (The Internet and information Technology in Modern Organizations), 2005.

[11] Aggelis V., Association Rules Model of e-Banking services, $5^{\text {th }}$ International Conference on Data Mining, Text Mining and their Business Applications, 2004.

[12] Aggelis V., E-Banking Integrated Data Utilization Platform WINBANK Case Study, Global Conference on Emergent Business Phenomena in the Digital Economy, 2006.

[13] Aggelis V., E-Banking: How Data Lead to Action, International Conference on Engineering and Mathematics, 2006.

[14] Aggelis V., e-Trans Association Rules Re-visited, 4th International Multiconference on Computer Science and Information Technology, 2006.

[15] Aggelis V., Offline Internet Banking Fraud Detection - 1st International Conference on Availability, Reliability and Security (ARES2006), Workshop "Dependability Aspects on Data Warehousing and Mining Applications", DAWAM, 2006.

[16] Aggelis V., The Lamp Example - Impact of Human Factor in KRIOS Model, XXIth Applied Stochastic Models and Data Analysis International Conference, 2007.

[17] Aggelis V., K.R.I.O.S., International Conference "Innovation, Entrepreneurship and Competitiveness in Balkan and Black Sea Countries", 2006.

[18] Aggelis V., The e-banking bible, New Technologies Publications, 2005. 